\title{
Selection during a selfing programme. I. The effects of a single round of selection
}

\author{
M. A. Cornish
}

School of Biological Sciences, The University of Birmingham, P.O. Box 363, Birmingham B15 2TT, U.K.

\section{INTRODUCTION}

Selection theory of quantitatively inherited characters has been developed to deal with outbreeding populations of various kinds (Falconer, 1981) and has been applied to a range of plant breeding programme designs, (see for example Hallauer and Miranda, 1981). Equations allow theoretical comparisons to be made between the responses expected from different designs of breeding programme and can also be used to predict the response a breeder is likely to obtain in practice. The theory, however, has been less extensively developed in the context of the selfing programmes commonly used to breed recombinant inbred lines of autogamous species, even though many major crop species are bred in this manner. In the absence of such a theoretical framework it is possible that many breeders of autogamous crop species fail to exploit fully the genetic variance available to them, because their resources are not allocated in the most efficient manner to the various stages of their breeding programmes.

This paper describes a theoretical study of the effects of a single round of selection at any generation during a selfing programme on both the mean and the genetical variance of the resultant inbred lines. The effect on the means of later generations has also been considered by others (Wricke and Weber, 1986). The effect of selection on the genetical variance has, however, only been examined in the context of outbreeding populations (Bulmer 1971, 1976). Selection during selfing presents a slightly different problem. In order to achieve an initial examination of these theoretical problems it has been necessary to consider a simplified selfing programme.

\section{THEORY}

\section{Selection responses}

In its simplest form, a selfing programme with a single round of selection can be described as follows. An initial cross is made between two inbred parents. Generations are then derived by single seed descent from the $F_{2}$, and selection for a single quantitative character is by truncation on the basis of individual scores in the $F_{2}$, or of family means in later generations. Following a single round of selection, lines derived from the selected individuals or from randomly chosen members of the selected families are descended by single seed descent to $F_{\infty}$, when the inbred lines are assessed. In this case the response differential, $R$, represents the difference between the mean of the inbreds 
produced by selection and the mean of the $F_{\infty}$ generation produced without selection. For simplicity I have assumed that environmental effects are constant over generations, that genotypes do not interact with environments, and that the character can be assessed on individual plants without the need for plots.

Given these simplifying assumptions the response to selection in the $\mathrm{F}_{2}$ takes the standard form:

$$
R_{\mathrm{F}_{2}}=i h_{\mathrm{N}^{2}} \sigma_{\mathrm{p}}
$$

where $i$ is the selection intensity, $h_{\mathrm{N}^{2}}$ is the narrow heritability of $F_{2}$ individuals and $\delta_{\mathrm{p}}$ is the phenotypic standard deviation of the $F_{2}$. Using the notation of Mather and Jinks (1982) this can be written as follows in the absence of epistasis:

$$
R_{\mathrm{F}_{2}}=i \frac{\frac{1}{2} D}{\left(\frac{1}{2} D+\frac{1}{4} H+E\right)^{1 / 2}} .
$$

Snape and Simpson (1984) present effectively the same equation with the inclusion of epistatic components. The response equations for family selection in later generations require the heritability and the phenotypic standard deviation appropriate to the families under selection. The denominator of the heritability is the phenotypic variance of the family means and, therefore, contains both rank 1 and rank 2 components. The numerator is the covariance between family means and the $\mathrm{F}_{\infty}$ generation. It also contains rank 1 and rank 2 components and can be seen to consist of the additive genetic components of the denominator. Thus the appropriate heritability of the $F_{3}$ family means is

$$
h_{\mathrm{F}_{3}}^{2}=\frac{\frac{1}{2} D+(1 / n)\left(\frac{1}{4} D\right)}{\frac{1}{2} D+\frac{1}{16} H+(1 / n)\left(\frac{1}{4} D+\frac{1}{8} H+E\right)}
$$

where $n$ is the family size. This gives the response equation in the absence of epistasis:

$$
R_{\mathrm{F}_{3}}=i \frac{\frac{1}{2} D+(1 / n)\left(\frac{1}{4} D\right)}{\left\lceil\frac{1}{2} D+\frac{1}{16} H+(1 / n)\left(\frac{1}{4} D+\frac{1}{8} H+E\right)\right\rceil^{1 / 2}} .
$$

Similarly, the response to selection of $F_{4}$ families derived from different $F_{2}$ plants is

$$
R_{\mathrm{F}_{4}}=i \frac{\frac{3}{4} D+(1 / n)\left(\frac{1}{8} D\right)}{\left\lceil\frac{3}{4} D+\frac{3}{64} H+(1 / n)\left(\frac{1}{8} D+\frac{1}{16} H+E\right)\right\rceil^{1 / 2}}
$$

or in general terms for the $N$ th generation

$$
\begin{aligned}
R_{\mathrm{F}_{N}}=i & \frac{\left(\left[1-\left(\frac{1}{2}\right)^{N-2}\right] D+(1 / n)\left[\frac{1}{2}\right)^{N-1}\right] D}{\left\{\left[1-\left(\frac{1}{2}\right)^{N-2}\right] D+\left[\left(\frac{1}{2}\right)^{N-}\left(\frac{1}{2}\right)^{2 N-2}\right] H\right.} \\
& \left.+(1 / n)\left\{\left[\left(\frac{1}{2}\right)^{N-1}\right] D+\left[\left(\frac{1}{2}\right)^{N}\right] H+E\right\}\right\}^{1 / 2}
\end{aligned}
$$

\section{Effects on the genetic variance}

Bulmer (1971) described the reduction in genetic variation in a randomly mating population following selection, which results from the linkage disequilibrium created by the selection. If the resultant population is then randomly mated without further selection, the variation is gradually restored to its original equilibrium value, assuming that the number of effective factors is infinite; the disequilibrium is halved in each generation in the absence of linkage. A similar reduction in genetic variation is to be expected from selection during a conventional selfing series from an $F_{1}$. In this case, however, no restoration takes place if selection is relaxed, because there is no random mating to reduce the disequilibrium. Instead the reduction in variation is inherited by all subsequent selfed generations, and further rounds of selection will reduce the genetic variation even further. Additional variation will however be created by further segregation during the selfing series.

These effects can be quantified by considering the simplified selfing programme described above. For example, consider the effect that selection in the $F_{2}$ generation has on the genetical variance of the subsequent $F_{\infty}$ generation. Call the additive genetic variance between the $\mathrm{F}_{2}$ individuals $\mathrm{V}_{\mathrm{A}}\left(\frac{1}{2} D\right.$ in the notation of Mather and Jinks (1982) in the absence of epistasis), and the phenotypic variance of the $F_{2}, V_{p}$. Following Bulmer's (1971) approach, the regression of the inbred lines $(Y)$ on their $F_{2}$ parents $(P)$ is given by

$$
\mathrm{Y}=a+b \mathrm{P}+e
$$

where $a$ is a constant, $e$ is the residual error, and the regression coefficient, $b$, is the narrow heritability of $F_{2}$ individuals. The variance of $Y$ is given by

$$
\operatorname{Var}(\mathrm{Y})=b^{2} \operatorname{Var}(\mathrm{P})+\operatorname{Var}(e)
$$

and

$$
\operatorname{Var}(e)=\operatorname{Var}(\mathrm{Y})-b^{2} \operatorname{Var}(\mathrm{P})
$$

If selection in the $F_{2}$ changes the phenotypic variance of the selected individuals from $V_{p}$ to $V_{p}+$ $d \mathrm{~V}_{\mathrm{p}}$, then the variance amongst the inbreds will become

$$
\begin{aligned}
\operatorname{Var}\left(\mathrm{Y}^{\prime}\right) & =b^{2}\left(\mathrm{~V}_{\mathrm{p}}+d \mathrm{~V}_{\mathrm{p}}\right)+\operatorname{Var}(e) \\
& =\operatorname{Var}(\mathrm{Y})+b^{2} d \mathrm{~V}_{\mathrm{p}} \\
& =\operatorname{Var}(\mathrm{Y})+\left(h^{2}\right)^{2} d \mathrm{~V}_{\mathrm{p}}
\end{aligned}
$$


The effect that selection has on the phenotypic variance of the selected $F_{2}$ individuals is given by

$$
d \mathrm{~V}_{\mathrm{p}}=-i(i-x) \mathrm{V}_{\mathrm{p}} \quad(\text { Cochran, 1951) }
$$

where $i$ is the intensity of selection, and $x$ is the standardised deviation of the point of truncation from the mean of the selected generation. The variance between the inbreds after selection therefore becomes

$$
\begin{aligned}
\operatorname{Var}\left(\mathrm{Y}^{\prime}\right) & =\operatorname{Var}(\mathrm{Y})-\left(h^{2}\right)^{2} i(i-x) \mathrm{V}_{\mathrm{p}} \\
& =\operatorname{Var}(\mathrm{Y})-h^{2} i(i-x) \mathrm{V}_{\mathrm{A}} \\
& =D-h^{2} i(i-x) \frac{1}{2} D .
\end{aligned}
$$

Following $F_{2}$ selection, the reduction in genetic variance appears in the inbreds as a reduction in the rank 1 variance, as defined by Mather and Jinks (1982), i.e. that portion of the variance which relates directly to the additive genetic variance of the $\mathrm{F}_{2}$ generation $\left(\frac{1}{2} D\right)$. For simplicitly this reduction has been considered in the $F_{\infty}$ generation, but the same reduction would be expected in the rank 1 variance in the $F_{3}$ generation onwards. The remainder of the genetic variance of the inbreds $\left(\frac{1}{2} D\right)$, that results from further segregation from the $F_{2}$ onwards, will be affected if the frequency of heterozygous loci is changed by selection. This effect has been assumed to be negligible in the case of selection in outbreeding populations (Falconer, 1981) and the same assumption will, for the time being, be made here. However, the validity of this assumption will be examined by simulation later in this paper.

This approach can also be applied to the selection of family means in later generations. In this case $V_{A}$ and $V_{p}$ refer to the additive genetic variance and the phenotypic variance of the family means, respectively, in the selected generation, these being the numerator and denominator of the appropriate heritability as described above. The reduction in variance again appears in subsequent generations in that portion of the total variance that relates to the variance between the selected families: additional variance generated by further segregation can again be assumed to be unaffected. If one or more rounds of selection have also been applied to earlier generations, then the expected reductions in $\mathrm{V}_{\mathrm{A}}, \mathrm{V}_{\mathrm{p}}$ and $h^{2}$ must be taken into account before the effects of later selection can be calculated.

\section{SIMULATION RESULTS}

\section{Effect on the $F_{7}$ mean}

Computer simulations provide a method of assessing the validity of response equations in the presence of realistic error variation and allow the investigation of additional complications that cannot easily be included in the deterministic approach. The program used here represents individual alleles of the genes controlling a quantitative character by binary digits. Using a pseudorandom number generator and bit-handling routines, segregation and recombination were simulated. The diploid genotypes generated were assigned genotypic scores according to the input values of additive and dominance effects. Environmental effects were simulated by a pseudo-random number generator which sampled a normal distribution.

Fig. 1 shows the results of simulations of singlestage selection in the $F_{2}, F_{3}$ and $F_{4}$ generations. In all cases, the simulated character was controlled by 20 independent genes of equal effect. The $F_{2}$ generation consisted of 100 individuals, and the $F_{3}$ and $F_{4}$ generations of 100 families, with 10 individuals per family, each family being derived from a different $F_{2}$ individual. Truncation selection for increased score was applied to individuals in the $F_{2}$, and to family means in the $F_{3}$ and $F_{4}$ generations. The proportion selected ranged from 1 to 75 per cent, and independent simulation runs were used for each selection intensity. The responses were assessed in the $F_{7}$ generation, in the absence of environmental variation, and are expressed as a standardised response by dividing by the additive genetic standard deviation $\left(D^{1 / 2}\right)$. Ten inbred lines were generated from each $F_{2}$ individual or from each $F_{3}$ or $F_{4}$ family. Each point on the graph represents the mean of 200 simulations in the case of $F_{2}$ selection, and 100 simulations otherwise.

The initial character was controlled only by additive genetic and environmental effects to provide a narrow heritability of 0.5 ; neither dominance nor epistasis were included. The curves represent the expected responses obtained from the appropriate response equations. A satisfactory agreement with expected was observed in all cases. Although the response equations include a component of dominance variation, they fail to take into account the additional influence of directional dominance effects. This can be best investigated by simulation. Two forms of dominance variation were simulated by adding dominance variation to the character described above; these were complete, positive dominance at all loci $(h=d)$ or complete, negative dominance at all loci $(h=-d)$. As is to be expected, because selection here was for increasing expression, complete positive dominance reduced the response observed, whilst complete negative dominance increased it. This effect was greatest when selection was most 


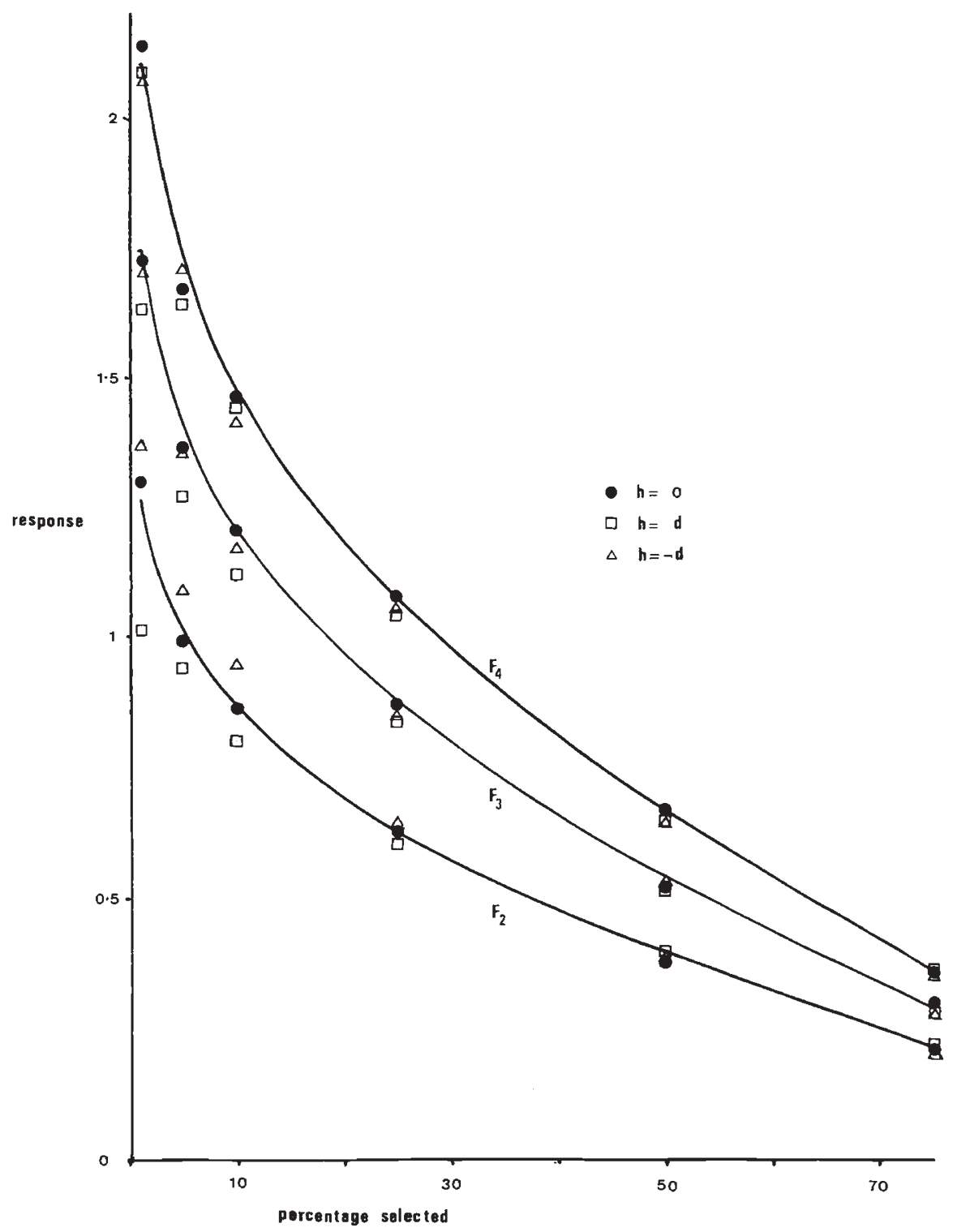

Figure 1 The effects of simulated single-stage selection in the $F_{2}, F_{3}$ and $F_{4}$ generations in terms of the standardised responses in the $F_{7}$ generation. The points represent the means of 200 simulations in the case of $F_{2}$ selection and 100 simulations otherwise. In all cases 20 independent genes of equal effect controlled a character with a narrow heritability of $0 \cdot 5$. Complete positive dominance, completely negative dominance and no dominance were simulated. The curves represent the expected responses.

intense, and was greater in the $F_{2}$ than the $F_{3}$. The average effects of even these extreme forms of dominance were not, however, large when compared with the expected response. By interpolation the effects of partial or ambidirectional dominance will be even less important.

\section{Effect on the genetic variance in the $F_{7}$}

The simulations described above were also used to examine the effect of $F_{2}$ and $F_{3}$ selection on the genetical variances of the resultant inbred lines. Ten $\mathrm{F}_{7}$ individuals were derived from each selected $F_{2}$ individual or from each selected $F_{3}$ family, by single seed descent. The inbreds were assessed without environmental error. This, therefore, permitted the genetic variation in the $F_{7}$ generation to be partitioned into a between $F_{2}$ parents item (the rank 1 variance) and a within $\mathrm{F}_{2}$ parents item; the two components of the expected mean squares were then estimated for each set of data. These will be referred to as the between groups and the 


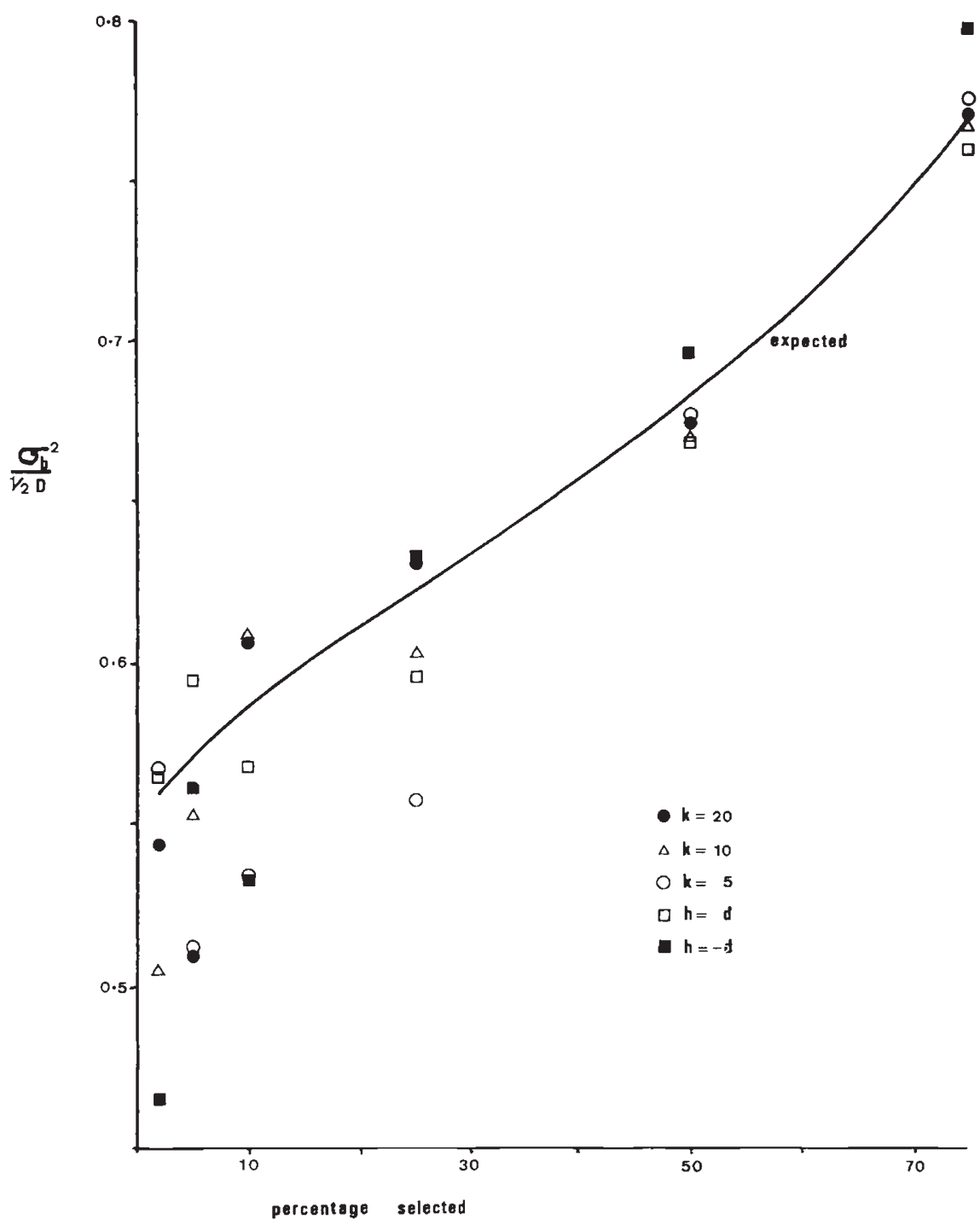

Figure 2 The effects of a single-stage selection in the $F_{2}$ generation on the variance between groups of $F_{7}$ lines, where each group is derived from a different $F_{2}$ individual. The points represent means of 200 simulations. All five characters have a narrow heritability of $0 \cdot 5$. Three additive characters were controlled by 20,10 and 5 genes of equal effect, with no dominance. Complete positive dominance and complete negative dominance were simulated for the 20 gene case.

within groups variances. $F_{2}$ simulations were repeated 200 times, and $F_{3}$ simulations 100 times. The different selection intensities were simulated independently.

In this case five quantitative characters were considered. In addition to the three characters described above, two further characters were generated by reducing the number of genes to 10 , and to 5 , in the absence of dominance, whilst keeping the additive genetic variance and the environmental variance constant.
The effects of selection on the between groups variance in the $\mathrm{F}_{7}$ generation are illustrated in figs 2 and 3 . In both cases the variance is expressed as a proportion of the variance expected in the absence of selection $\left(\frac{1}{2} D\right)$. The line represents the variance expected from the theory presented above, assuming no dominance and an infinite number of genes. The observed results are presented for each of the five characters. As the proportion selected decreased, the degrees of freedom of the between groups item also decreased and the 


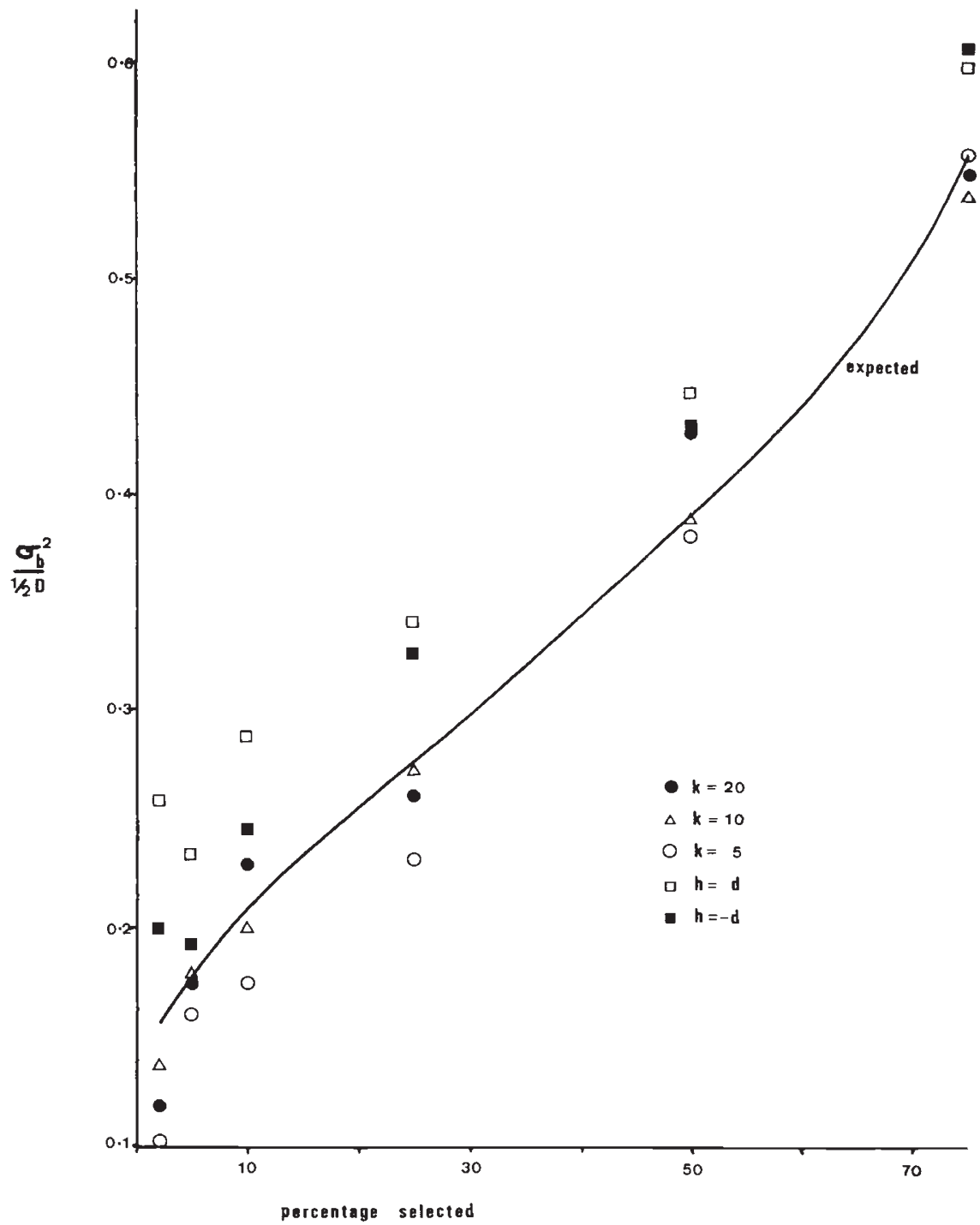

Figure 3 The effects of single-stage selection in the $F_{3}$ generation on the variance between groups of $F_{7}$ lines, where each group is derived from a different $F_{3}$ family. The points present the means of 100 simulations. The characters are the same as in fig. 2 .

errors on the estimates increased. Given a heritability of 0.5 in the $F_{2}$, and families of 10 individuals in the $F_{3}$, selection is relatively effective and therefore the variance is expected to be reduced quite considerably. Bearing in mind the variability of variance estimates, the means of the observed variances followed the expected lines reasonably closely. With $F_{2}$ selection there were no clear differences between the five characters. With more effective selection in the $F_{3}$, however, slight differences became apparent. Thus in the presence of dominance variation (positive or negative) a slightly larger variance than expected was con- stantly observed. With only five genes segregating, the observed variances were reduced slightly at higher selection intensities. Compared with the errors on variances in practice these biases are small and are only likely to be observed in a theoretical study using highly repeated simulations. The effects of selection on the variances in the generation following selection were also studied and similar agreements with expected were found.

In figs 4 and 5 responses to selection of the within group variances can be seen. These are also expressed as a proportion of the value expected 


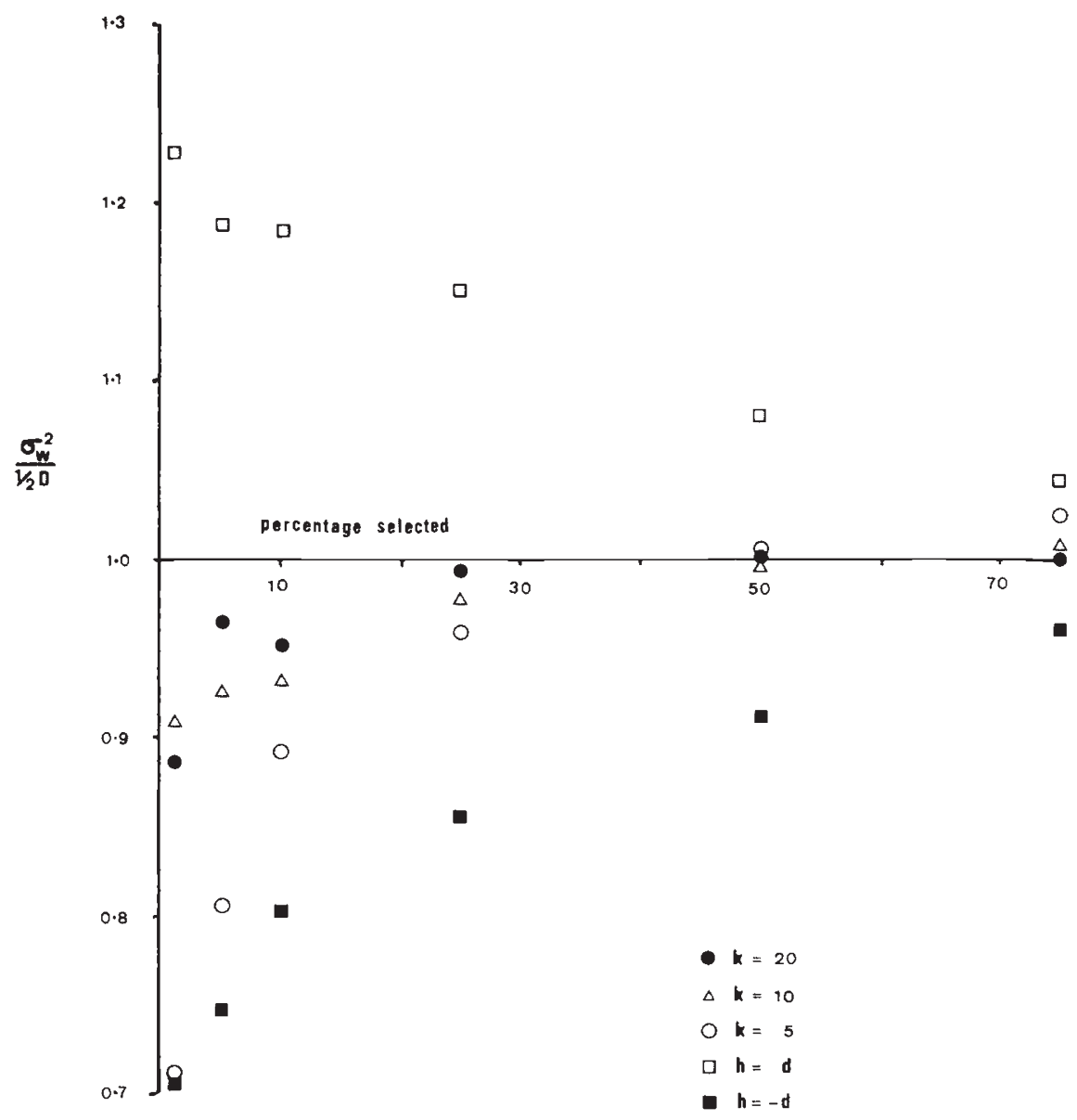

Figure 4 The effects of single-stage selection in the $F_{2}$ on the variance within groups of $F_{7}$ lines. The points are the means of 200 simulations. The characters are the same as in fig. 2.

in the absence of selection, $\frac{1}{2} D$, which is represented by the horizontal axis. In both cases the additive genetic character controlled by 20 genes followed the horizontal axis closely, except at higher selection intensities when a small reduction in variance was found. This effect became more marked as the number of genes fell. This can be interpreted as revealing a tendency for slightly fewer heterozygotes to be selected than would be expected in the absence of selection. Complete, positive dominance led to an increase in the within group variance as selection increased the frequency of heterozygotes a little; negative dominance produced the opposite effect. Biases caused by complete dominance were more important in the $F_{2}$ than the $F_{3}$, as is to be expected, but reducing the number of genes had a greater effect following $F_{3}$ selection. Biases caused by partial dominance will be smaller than those observed here, whilst ambidirectional dominance will produce no overall effect.

\section{OPTIMUM DESIGNS}

Response equations can be used to compare different designs of breeding programme and when all possible designs are examined, within a defined set of circumstances, the optimum design, that which maximizes the expected final response, can be determined. In the case of single-stage selection programmes, for a given character and a given number of plants, an optimum design can be determined for selection at each generation. This permits a comparison of the effectiveness of selection at each generation, and will also allow single-stage selection designs to be compared with optimum 


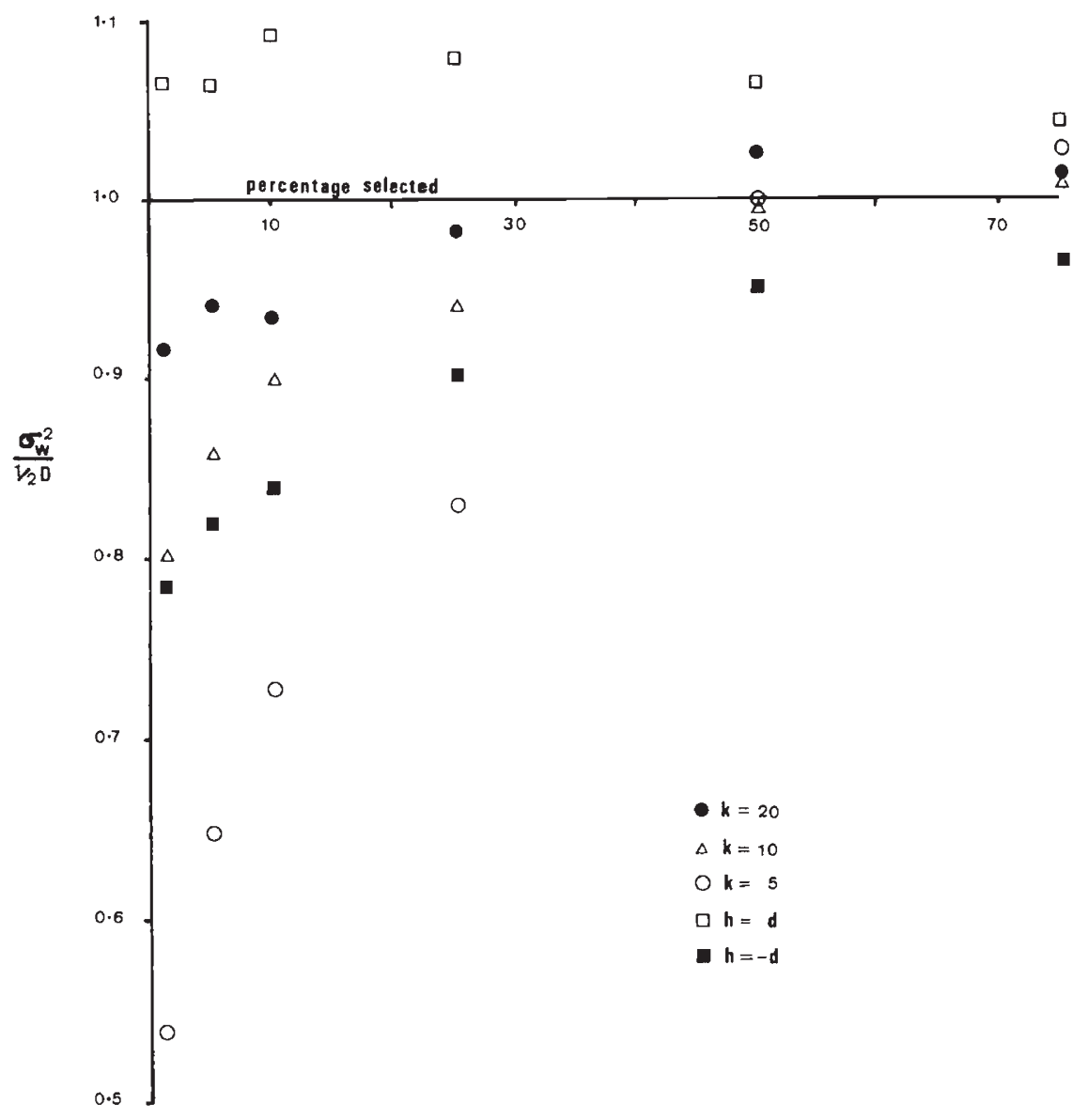

Figure 5 The effects of single-stage selection in the $F_{3}$ on the variance within groups of $F_{7}$ lines. The points are the means of 100 simulations. The characters are the same as in fig. 2.

multi-stage designs that utilize the same resources (see following paper).

To illustrate the approach taken, consider the simplified selfing programme described above. If we impose a maximum limit of 1000 plants in the selected generation, and we aim to generate a single inbred $\left(\mathrm{F}_{7}\right)$ line from a single round of selection, then the optimum design for $F_{2}$ selection is fixed: the best approach is to derive the inbred line from the highest scoring of $100 \mathrm{~F}_{2}$ plants. In later generations a number of different designs are possible, differing in the number of families and the number of individuals per family in the selected generation (assuming all families to be of equal size in each generation). The optimum design is dependent upon the properties of the character under selection, particularly the $F_{2}$ narrow heritability and, in the absence of dominance and epistasis, will depend entirely on this narrow heritability. A com- puter program, incorporating the appropriate response equations, was used to calculate the expected responses from all possible designs within the limitations imposed, and thus to determine the optimum designs at each generation for a range of narrow heritabilities. For simplicity, dominance and epistasis were assumed to be absent. The selection intensity, $i$, used in these equations was obtained from tables from Harter (1961) when selection was from a population of 100 or less and from Burrows' (1972) approximation when the population was greater than 100 .

The optimum designs for single-stage selection from generation $F_{2}$ to $F_{6}$ with 1000 plants in the selected generation are presented in table 1 , as the top line within each category. The expected result is expressed as a standardized response. As is to be expected, the optimum response decreases as the heritability decreases, and increases as 
Table 1 Optimum single-stage selection designs for a range of narrow heritabilities. $m$ is the number of families, $n$ is the family size, $R$ is the expected standardised selection response. Within each category the top line is the optimum design with a maximum of 1000 plants in the selected generation, the bottom line is with 1000 plants between the $F_{2}$ and $F_{6}$ inclusive.

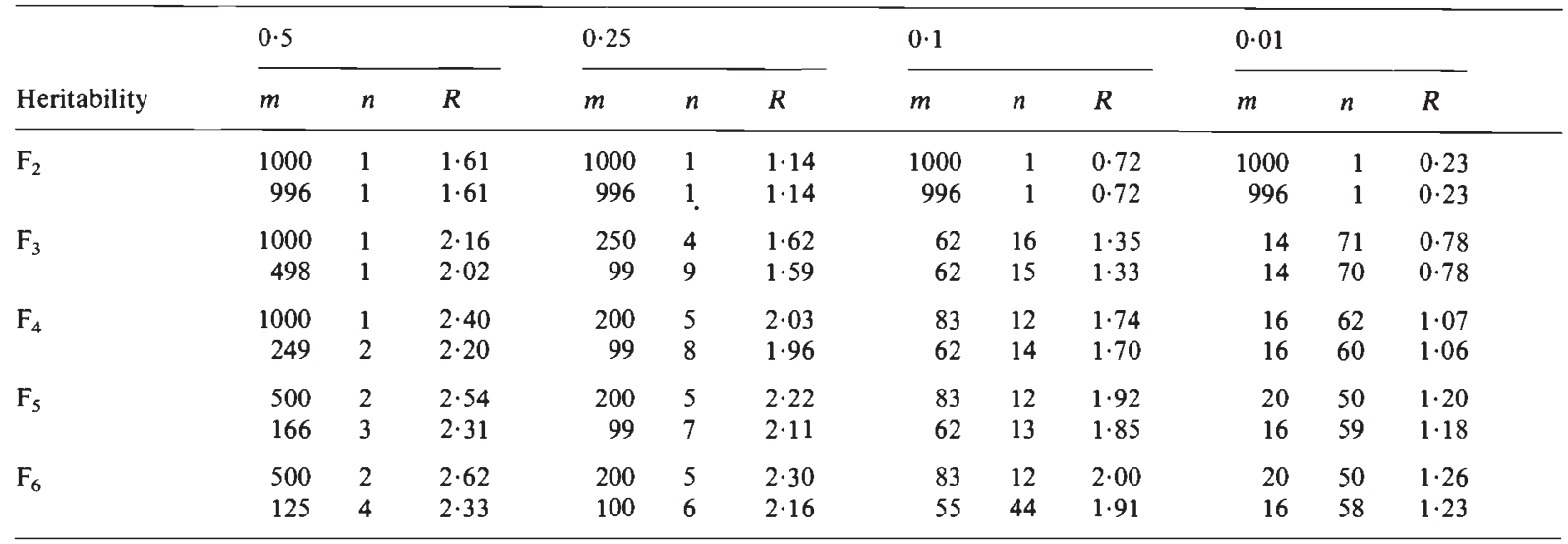

homozygosity increases. As Pooni and Jinks (1985) concluded, the benefits of delaying selection are greater for characters of low heritability. Large families are only of value when the heritability is low and individual selection is preferable to family selection in the $F_{3}$ and $F_{4}$ generations when the heritability is high.

It can, however, be argued that these comparisons between generations are unfair. For example, selection of $1000 \mathrm{~F}_{4}$ families would also require $1000 \mathrm{~F}_{2}$ plants and $1000 \mathrm{~F}_{3}$ plants to be grown. Such a programme would therefore require three times the number of plants required by the $F_{2}$ selection programme. An alternative approach is to include within the limit of 1000 plants all the plants required, from generations $\mathrm{F}_{2}$ to $\mathrm{F}_{6}$ inclusive to produce a single $\mathrm{F}_{7}$ line. In this case the optimum $\mathrm{F}_{2}$ design requires the selection of the best plant from $996 \mathrm{~F}_{2}$ plants, the remaining four being used to descend the selected line to $F_{7}$. These new optimum designs are shown as the second line within each category in table 1 . Taking into account plants in the unselected generations alters the optimum designs in many cases, but reduces the expected responses only slightly. In table 2 the expected responses for each generation are compared with the responses from selection in the $F_{6}$, which can be considered to represent the optimum single seed descent programme. In this very simplified example, early generation selection can be expected to give a reasonable response when the heritability is high, but delaying selection until the $F_{6}$ gives only a small improvement over the $F_{4}$ or $\mathrm{F}_{6}$ unless the heritability is very low.

It is also informative to investigate these optimum designs by simulation. Table 3 shows the
Table 2 Expected responses from optimum single-stage selection designs relative to the response expected in the $F_{6}$. Top line and bottom line within each category as for table 1

\begin{tabular}{lllll}
\hline$h_{n}^{2}$ & 0.5 & 0.25 & 0.1 & 0.01 \\
\hline $\mathrm{F}_{2}$ & 0.61 & 0.49 & 0.36 & 0.18 \\
& 0.69 & 0.53 & 0.38 & 0.18 \\
$\mathrm{~F}_{3}$ & 0.82 & 0.71 & 0.67 & 0.62 \\
& 0.86 & 0.73 & 0.70 & 0.63 \\
$\mathrm{~F}_{4}$ & 0.92 & 0.88 & 0.87 & 0.85 \\
& 0.94 & 0.91 & 0.89 & 0.85 \\
$\mathrm{~F}_{5}$ & 0.97 & 0.96 & 0.96 & 0.95 \\
& 0.99 & 0.98 & 0.97 & 0.95 \\
\hline
\end{tabular}

results of simulations of the optimum $\mathrm{F}_{6}$ selection programmes, when the whole programme is limited to a maximum of 1000 plants. In the simplest case 20 independent loci were simulated in the absence of dominance or epistasis. As before the mean response from 200 simulations agrees well with the expected value. The variances of the final responses can be seen to increase as the heritability falls. Even at the highest heritability fewer than 15 of the 20 genes were on average fixed for the increasing allele.

Table 3 also shows the effect on the final response of reducing the number of genes, $k$, controlling the character, whilst holding the total genetic variation constant. As $k$ was reduced the observed response fell, although the effect was relatively small. When epistasis was added to the character, on the other hand, a marked departure from expected was observed. In the cases simulated, a relatively small amount of epistasis 
Table 3 Results of 200 simulations of the optimum $F_{6}$ selection designs ( 1000 plants maximum between the $F_{2}$ and $F_{6}$ ).

\begin{tabular}{|c|c|c|c|c|c|}
\hline & $\begin{array}{l}h_{n}^{2} \\
\text { Expected } \\
\text { mean }\end{array}$ & $\begin{array}{l}0 \cdot 5 \\
2 \cdot 34\end{array}$ & $\begin{array}{l}0 \cdot 25 \\
2 \cdot 16\end{array}$ & $\begin{array}{l}0 \cdot 1 \\
1 \cdot 91\end{array}$ & $\begin{array}{l}0 \cdot 01 \\
1 \cdot 24\end{array}$ \\
\hline$k=20$ & $\begin{array}{l}\text { Mean } \\
\text { Variance } \\
\boldsymbol{A A} \\
\boldsymbol{A} a\end{array}$ & $\begin{array}{r}2 \cdot 24 \\
0 \cdot 29 \\
14.90 \\
0.25\end{array}$ & $\begin{array}{r}2 \cdot 18 \\
0 \cdot 27 \\
14 \cdot 74 \\
0 \cdot 26\end{array}$ & $\begin{array}{r}1.97 \\
0.35 \\
14.25 \\
0.30\end{array}$ & $\begin{array}{r}1.20 \\
0.69 \\
12.50 \\
0.38\end{array}$ \\
\hline$k=10$ & $\begin{array}{l}\text { Mean } \\
\text { Variance } \\
\boldsymbol{A A} \\
\boldsymbol{A} a\end{array}$ & $\begin{array}{l}2 \cdot 26 \\
0 \cdot 22 \\
8 \cdot 51 \\
0 \cdot 13\end{array}$ & $\begin{array}{l}2 \cdot 05 \\
0 \cdot 30 \\
8 \cdot 18 \\
0 \cdot 12\end{array}$ & $\begin{array}{l}1 \cdot 79 \\
0 \cdot 33 \\
7 \cdot 77 \\
0 \cdot 14\end{array}$ & $\begin{array}{l}1.20 \\
0.56 \\
6.76 \\
0 \cdot 17\end{array}$ \\
\hline$k=5$ & $\begin{array}{l}\text { Mean } \\
\text { Variance } \\
\boldsymbol{A A} \\
\boldsymbol{A} a\end{array}$ & $\begin{array}{l}2.09 \\
0 \cdot 10 \\
4 \cdot 82 \\
0.05\end{array}$ & $\begin{array}{l}1.93 \\
0.21 \\
4.65 \\
0.03\end{array}$ & $\begin{array}{l}1.78 \\
0.24 \\
4.45 \\
0.07\end{array}$ & $\begin{array}{l}1 \cdot 23 \\
0.46 \\
3 \cdot 84 \\
0.07\end{array}$ \\
\hline $\begin{array}{l}i \\
\text { positive }\end{array}$ & $\begin{array}{l}\text { Mean } \\
\text { Variance } \\
\boldsymbol{A A} \\
\boldsymbol{A} a\end{array}$ & $\begin{array}{r}2 \cdot 69 \\
0 \cdot 31 \\
15 \cdot 12 \\
0 \cdot 28\end{array}$ & $\begin{array}{r}2.55 \\
0.55 \\
14.88 \\
0.30\end{array}$ & $\begin{array}{r}2 \cdot 17 \\
0.65 \\
14 \cdot 17 \\
0.35\end{array}$ & $\begin{array}{r}1.42 \\
0.92 \\
12.79 \\
0.35\end{array}$ \\
\hline $\begin{array}{l}i \\
\text { negative }\end{array}$ & $\begin{array}{l}\text { Mean } \\
\text { Variance } \\
A A \\
A a\end{array}$ & $\begin{array}{r}1.90 \\
0.12 \\
14.74 \\
0.33\end{array}$ & $\begin{array}{r}1 \cdot 70 \\
0 \cdot 19 \\
14.22 \\
0.20\end{array}$ & $\begin{array}{r}1.60 \\
0.23 \\
13.86 \\
0.29\end{array}$ & $\begin{array}{r}1.09 \\
0.38 \\
12.40 \\
0.35\end{array}$ \\
\hline
\end{tabular}

The mean and variance of the observed standardized responses are given along with the average number of genes fixed for the increasing allele, and the average number of heterozygous genes. The five characters are described in the text.

between homozygous loci $(I /(D+I)=0.01)$ was distributed equally across all pairwise combinations of 20 unlinked genes. Whilst the epistatic variance was small, this kind of epistasis, nevertheless, generated considerable skewness amongst the inbred lines being selected: this led to a better response than expected with complementary epistasis $(+i)$, but a worse response than expected with duplicate epistasis $(-i)$.

\section{DISCUSSION}

In order to compare different kinds of selection it is necessary, first, to determine the most efficient form of each kind of selection in a given set of circumstances. A fair comparison can then be made between the different optimum designs. This has been achieved here for a set of simplified cases. The effectiveness of selection in early and later generations of a selfing programme, which has been discussed in the literature (Weber, 1984; Jinks and Pooni, 1981), has been quantified and compared. It can be seen that, given the simplifying assumptions described, early generation selection of individual plants can provide a large proportion of the response expected from a single round of selection in the $\mathrm{F}_{6}$ if the heritability of the character is relatively high. The response expected from a single round of selection in the $\mathrm{F}_{5}$ and in some cases the $\mathrm{F}_{4}$ can be almost as good as that expected from $\mathrm{F}_{6}$ selection.

The value of using computer simulations to supplement deterministic theory is illustrated by the study of the effects of directional dominance, which cannot be predicted from the response equations. The two forms of dominance simulated produced deviations in the mean response in the expected direction. Even following extreme selection in the $\mathrm{F}_{2}$, these deviations were relatively small compared with the sampling error. These deviations will become even less important at heritabilities lower than the 0.5 simulated here and when dominance is less than complete or is ambidirectional. These results reveal that it is the high degree of heterozygosity in the early generations, rather than the expression of dominance, that leads to a reduced response compared with later generations.

Simulations were also used to investigate the importance of the number of independent genes controlling a character. When this number was reduced from 20 to 5 , a small reduction in the average response was observed from the most effective selection programmes. When so few genes were segregating and the heritability was $0 \cdot 5$, the optimum design in table 1 fixed increasing alleles at all five loci 82 per cent of the time. If a character is segregating at only a few loci the result expected from an effective selection programme may be greater than the mean of the best possible inbred line. In contrast, when 20 genes were simulated the best possible inbred was not obtained.

Epistasis can generate non-normality in the distribution of inbred lines, particularly in the form of skewness (Pooni, Jinks and Cornish, 1977). Complementary epistasis $(+i)$ generates positive skewness, so that when selecting amongst $F_{6}$ families for increasing expression one is selecting in the elongated upper tail of the distribution. Because selection theory assumes normality, one is likely to select, from this tail, individuals more extreme than expected, and therefore observe a better response than expected. This was revealed by simulations, which also showed that the converse is true with duplicate epistasis.

Turning now to the effect of selection on genetic variances, it is clear that the adaptation of Bulmer's approach provided a satisfactory description of the main source of variance reduction in the simu- 
lated selection programme. The additive genetic variation between the individuals or families under selection was reduced considerably by effective selection. Furthermore, unlike that in outbreeding programmes, this reduction was not restored during the selfing series but was inherited by subsequent generations. The five quantitative characters that were simulated behaved in a very similar manner.

The computer simulations were particularly valuable in revealing the effect selection had on genetic variation generated by segregation in selected individuals and their progeny. When complete, unidirectional dominance was acting in the same direction as selection, there was a tendency in the early generations to select individuals and families which were slightly more heterozygous than would be expected in the absence of selection and this tended to inflate the additional variation produced by segregation. When the number of effective factors was small, or when selection was for the recessive character, then intense selection was at the expense of heterozygotes, so that the new variance generated was reduced. When, however, selection was less intense and, hence, less effective in the early generations, when dominance was in the direction of the selection applied, or when the number of effective factors was not small, the biases from this source were relatively minor compared with the predictable reduction in genetic variation between the selected families. Furthermore, in later generations the contribution made by the additional variance generated by segregation to the total genetic variation declines considerably. Thus, for most characters the theory presented described the major part of the reduction in genetic variation resulting from selection under these circumstances. As in the case of outbreeding programmes, it may be adequate to assume that the variance generated by further segregation is unaffected by selection.

The programmes designed and simulated here are, of course, very simplified. Many of the complications that a plant breeder faces in practice have been omitted in order to achieve the objectives of this preliminary study of selection theory. Nevertheless, the results show that the response to a single round of simulated selection can be predicted, and that an approximate description of the reduction in genetic variance as a result of selection is possible. Experimental evidence is, of course, required from a range of species to determine whether these effects are found in practice.

Acknowledgement I am indebted to Dr M. J. Lawrence for his advice during the preparation of this article.

\section{REFERENCES}

BULMER, M. G. 1971. The effect of selection on genetic variability. Am. Nat., 105, 201-211.

BULMER, M. G. 1976. The effects of selection on genetic variability: a simulation study. Genet. Res., 28, 101-117.

BURROWS, P. M. 1972. Expected selection differentials for directional selection. Biometrics, 28, 1091-1100.

COCHRAN, W. G. 1951. Improvement by means of selection. Proc. Second Berkeley Symp. Math. Stat. Prob. 449-470.

FALCONER, D. S. 1981. Introduction to Quantitative Genetics, 2nd edn. Longman, London.

HALLAUER, A. R. AND MIRANDA, J. B. 1981. Quantitative Genetics and Maize Breeding. Iowa State University Press, Ames, U.S.A.

HARTER, H. L. 1961. Expected values of normal order statistics. Biometrics, 48, 151-165.

JINKS, J. L. AND POONI, H. S. 1981. Comparative results of selection in the early and late stages of an inbreeding programme. Heredity, 46, 1-7.

MATHER, K. AND JINKS, J. L. 1982. Biometrical Genetics, 3rd edn. Chapman and Hall, London.

POONI, H. S. AND JINKS, J. L. 1985. Retrospective selection and sources of superior inbreds amongst pedigree inbred families of Nicotiana rustica. Heredity, 54, 255-260.

POONI, H. S., JINKS, J. L. AND CORNISH, M. A. 1977. The causes and consequences of non-normality in predicting the properties of recombinant inbred lines. Heredity, 38, 329338.

SNAPE, J. W. AND SIMPSON, E. 1984. Early generation selection and rapid generation advancement methods in autogamous crops. Proceedings of the 10th Congress of EUCARPIA, Pudoc, Wageningen, pp. 82-86.

WEBER, W. E. 1984. Selection in early generations. Proceedings of the 10th Congress of EUCARPIA, Pudoc, Wageningen, pp. 72-81.

WRICKE, G. AND WEBER, W. E. 1986. Quantitative Genetics and Selection in Plant Breeding. Gruyter, Berlin. 